\title{
Photon Beamline Frontends for the PETRA III Extension Project
}

\author{
H.Schulte-Schrepping ${ }^{\text {a) }}$, M.Hesse, M.Degenhardt, H.Krüger, R.Peters, H.B.Peters, \\ B.Steffen
}

\author{
Deutsches Elektronen-Synchrotron DESY, Notkestraße 85, 22607 Hamburg, Germany \\ ${ }^{a)}$ Corresponding author: horst.schulte-schrepping@desy.de
}

\begin{abstract}
The photon beamline frontend design for the new insertion device (ID) beamlines of the PETRA III extension project will be presented. The design is based on the concepts developed for the photon beamline frontends at PETRA III. This generic design approach minimized the number of specialized components for all beamlines. The girder concept with kinematic mounts at each girder allowed a fast and reliable installation phase. The extension beamlines are located in two new additional buildings. There will be 4 sectors with two undulator IDs in each sector with a canting angle of 20mrad between the insertion devices. Additionally, two straight sections and a bending magnet chamber will be modified: one straight section will be transformed to a side station sector, the straight section with the 40m long damping wiggler will be used as a hard X-ray source, and the bending magnet will serve as a soft-X-ray source.
\end{abstract}

\section{GENERIC FRONTEND DESIGN CONSIDERATIONS}

The basic design considerations of all frontends at the PETRAIII extension project follow the generic beamline concept of the PETRA III project [1, 2]. In the course of this project 14 undulator insertion device (ID) frontends have been designed and installed. All beamlines are now in user operation in the PETRA III Max von Laue experimental hall with several experimental stations. The main feature of all frontends is the grouping and mounting of functional systems, i.e. masks, screen units, slit-systems, shutters, on a set of granite girders. The systems are mounted, interconnected, wired and vacuum conditioned on their respective girders outside of the tunnel in a preparation area under clean room environments. Each girder is mounted in the tunnel onto two columns which are aligned by the DESY survey group. The interface between the columns and the girders is a kinematic mount system.

The next step in the development of PETRA III as a synchrotron radiation source is the PETRA III extension project $[3,4]$. The project will add up to 10 further insertion device beamlines and one bending magnet beamline in order to provide and enhance the PETRA III user operation by experimental capabilities currently not available. The new frontend designs and components will be presented and the boundary conditions to the operation, i.e. the common vacuum system in the side station sector and the fixed gap operation of the damping wiggler section, will be discussed.

In the course of the development for the new frontends for the PETRA III extension project, some components were upgraded. Cooled CVD diamond screens allow to image the white beam footprint also at $100 \mathrm{~mA}$ ring current as compared to $1 \mathrm{~mA}$ in the original uncooled design. A modified high power filter system was introduced to be able to also image the white beam footprint at the installed CVD diamond filter.

Common to all frontends at PETRA III is a permanent dump magnet to protect against particles travelling along the frontend. This is a measure against the unlikely case that a bending magnet upstream of a frontend gradually or fully fails and the top-up operation still replenishes the current in the machine for some time until the machine protection shuts down the operation of the machine. 


\section{CANTED UNDULATOR FRONTEND}

All frontends in the canted sectors of the extension project will have a canting angle of 20mrad in the horizontal plane between the two undulator beamlines in the sector. The main difference to the generic PETRA III canted beamline design is the larger canting angle, which is enlarged from $5 \mathrm{mrad}$ to $20 \mathrm{mrad}$. The gained horizontal distance between the two beamlines allows for the installation of a separate girder system for each beamline. The overall distance from the source to the optics hutch grows from $30 \mathrm{~m}$ to $45 \mathrm{~m}$ due to the different topology of the machine in the tunnel. The camera readout at the cooled CVD-diamond screens, filters and windows at the optics hutch entrance allow for a setup of the canting angle and alignment of the two white photon beams along the frontends with the full stored current in the machine. Now, possible temperature effects induced by the different machine conditions due to the alignment operation at $1 \mathrm{~mA}$ and the user run at $100 \mathrm{~mA}$ cannot anymore cause errors in the photon beam position.

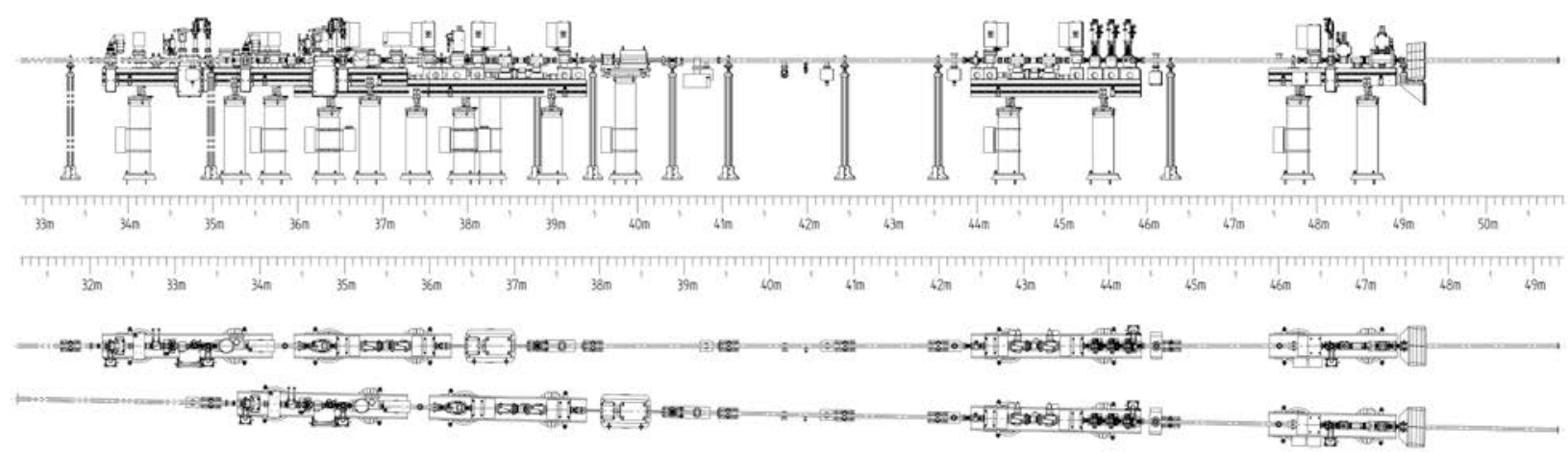

FIGURE 1. Layout of the 20mrad canted undulator front end. Top: side view of both front ends. Bottom: top view showing the angular separation of $20 \mathrm{mrad}$. The scales show the distance to the respective undulator source. Only the girder based section ending at the storage ring shielding wall, and the vacuum tube crossing the wall into the optics hutch are shown.

\section{STRAIGHT SECTION FRONTENDS}

This chapter describes the basic design considerations of the two straight sections of the PETRA III extension project. The straight section occupied by the $40 \mathrm{~m}$ long damping wiggler (DW) will be used as a hard X-ray source. The other straight section will be transformed to a side station sector served by two insertion devices with an angular separation of $1 \mathrm{mrad}$ in the horizontal plane. The on-axis station will use an in-vacuum undulator (IVU) and the side station will use a standard $2 \mathrm{~m}$ long ID.

\section{Damping Wiggler Frontend}

The 40m long damping wiggler located in a long straight section of PETRA III, together with a second identical wiggler at a different location, is installed to reduce the emittance down to the 1 nmrad level. The last photon absorber chamber of the machine vacuum system in the damping wiggler section will serve as the exit chamber for the new photon beamline frontend. The original design of the absorber chamber [5] will be modified into an exit chamber by introducing a cut-out of $3 \mathrm{~mm} \times 2 \mathrm{~mm}$ (horizontal x vertical) in size. Since the damping wiggler [6] is a fixed gap design, a mask system and a high-power shutter are needed to protect the first vacuum valve in the frontend. All other downstream components, i.e. slit systems, dump magnet, filter units, and Bremsstrahlung shutter are generic PETRA III systems. 


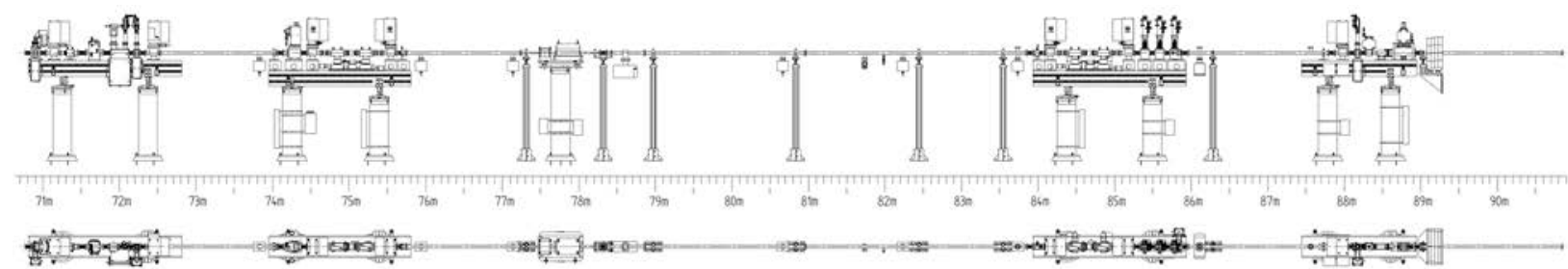

FIGURE 2. Layout of the damping wiggler front end. The girder based section downstream of the 40m long damping wiggler source is shown beginning at $71 \mathrm{~m}$ distance from the source. At the right hand side the vacuum tube crossing the shielding wall into the optics hutch is shown.

\section{Side Station Frontend}

The side station frontend will transport an on-axis white beam from a $4 \mathrm{~m}$ in-vacuum undulator together with the white beam of a $2 \mathrm{~m}$ insertion device [7] which is canted by $1 \mathrm{mrad}$ in the horizontal plane. Both photon beam transports in the side station frontend share a common vacuum system, but still the generic frontend components, i.e. masks, shutters and high-power slit systems are used. Due to the space constraint given by the $1 \mathrm{mrad}$ angular separation of both beamlines in the frontend, the components are distributed over a length of $18 \mathrm{~m}$ and 8 granite girders. The two vacuum systems are pumped individually at the components, and additionally are connected by common larger pumping vessels distributed along the frontend. The only new designed beamline component is the main Bremsstrahlung shutter. Here, collimation and shutter systems for both frontends are installed into a common vacuum vessel and still allow a separate operation of both beamlines.

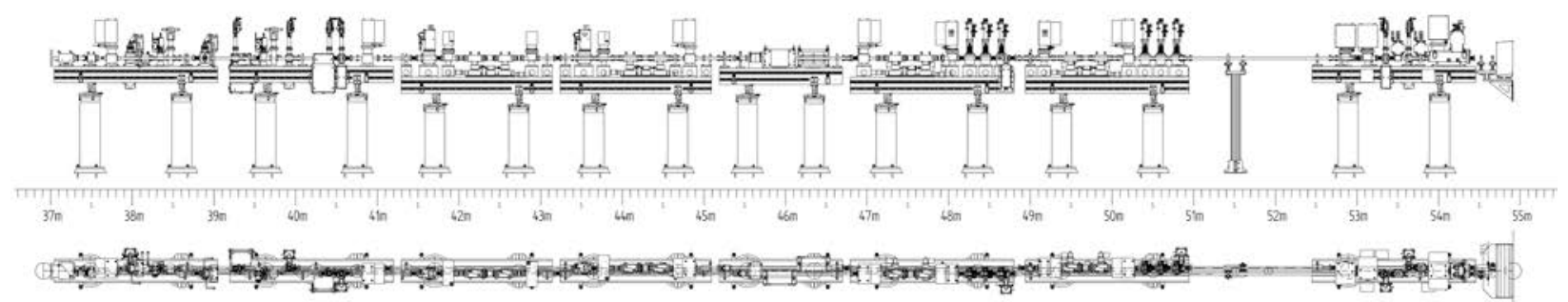

Figure 3. Layout of the side station frontend. The two photon beamlines with an angular separation of 1 mrad are mounted on a common granite girder system. The vacuum systems are separated at the generic beamline components and connected in between by common pumping vessels. The white beam enters the shown frontend part from the left side at $37 \mathrm{~m}$ distance from the source and crosses the shielding wall into the optics hutch at the right.

\section{ACKNOWLEDGMENTS}

The authors thank the involved DESY infrastructure groups and beamline staff for their input during the design phase of the PETRA III extension project. 


\section{REFERENCES}

1. U. Hahn, H.B. Peters, R. Röhlsberger, H. Schulte-Schrepping, "The Generic Beamline Concept of the PETRA III undulator beamlines,” AIP Conference Proceedings 879, (2006), pp. 539-542.

2. M. Degenhardt, H.B. Peters, U. Hahn, J. Heuer, M. Hesse, H. Schulte-Schrepping, "Status of PETRA III photon beamline frontends and optical systems,” J.Phys.:Conf. Ser. 4250042005.

3. W. Drube, et.al.,"The PETRA III Extension,” these proceedings.

4. K. Balewski, M. Bieler, J. Keil, A. Kling, G.K. Sahoo, R. Wanzenberg, "PETRA III upgrade,“ Proceedings of IPAC2011, San Sebastián, Spain, 2011, pp. 2948-2950.

5. M. Tischer, K. Balewski, M. Seidel, L. Yongjun, P. Vobly, V. Kuzminykh, A. Krasnov, K. Zolotariov, E. Levichev, "Status of the PETRA III Damping Wigglers," Proceedings of EPAC 2006, Edinburgh, Scotland, 2006, pp. 3565-3567.

6. M. Tischer, K. Balewski, A. Batrakov, I. Ilyin, D. Shichkov, A. Utkin, P. Vagin, P. Vobly, "Damping Wigglers at the PETRA III Light Source,” Proceedings of EPAC08, Genoa, Italy, 2008, pp. 2317-2319.

7. A. Schöps, P. Vagin, M. Tischer, "Properties of the Insertion Devices for PETRA III and its Extension," these proceedings 